\title{
Prediction of lung emphysema in COPD by spirometry and clinical symptoms: results from COSYCONET
}

Christina Kellerer ${ }^{1,2^{*}} \mathbb{0}$, Rudolf A. Jörres ${ }^{2}$, Antonius Schneider ${ }^{1}$, Peter Alter ${ }^{3}$, Hans-Ulrich Kauczor ${ }^{4,5}$, Bertram Jobst ${ }^{4,5}$, Jürgen Biederer ${ }^{4,5,6,7}$, Robert Bals ${ }^{8}$, Henrik Watz ${ }^{9}$, Jürgen Behr ${ }^{10}$, Diego Kauffmann-Guerrero ${ }^{10}$, Johanna Lutter ${ }^{11}$, Alexander Hapfelmeier ${ }^{1}$, Helgo Magnussen ${ }^{9}$, Franziska C. Trudzinski ${ }^{12}$, Tobias Welte ${ }^{13}$, Claus F. Vogelmeier ${ }^{3}$ and Kathrin Kahnert ${ }^{10}$

\begin{abstract}
Background: Lung emphysema is an important phenotype of chronic obstructive pulmonary disease (COPD), and CT scanning is strongly recommended to establish the diagnosis. This study aimed to identify criteria by which physicians with limited technical resources can improve the diagnosis of emphysema.

Methods: We studied 436 COPD patients with prospective CT scans from the COSYCONET cohort. All items of the COPD Assessment Test (CAT) and the St George's Respiratory Questionnaire (SGRQ), the modified Medical Research Council (mMRC) scale, as well as data from spirometry and CO diffusing capacity, were used to construct binary decision trees. The importance of parameters was checked by the Random Forest and AdaBoost machine learning algorithms.
\end{abstract}

Results: When relying on questionnaires only, items CAT 1 \& 7 and SGRQ 8 \& 12 sub-item 3 were most important for the emphysema- versus airway-dominated phenotype, and among the spirometric measures $F E V_{1} / F V C$. The combination of CAT item $1(\leq 2)$ with $\mathrm{mMRC}(>1)$ and FEV $1 / F V C$, could raise the odds for emphysema by factor 7.7 . About $50 \%$ of patients showed combinations of values that did not markedly alter the likelihood for the phenotypes, and these could be easily identified in the trees. Inclusion of CO diffusing capacity revealed the transfer coefficient as dominant measure. The results of machine learning were consistent with those of the single trees.

Conclusions: Selected items (cough, sleep, breathlessness, chest condition, slow walking) from comprehensive COPD questionnaires in combination with $\mathrm{FEV}_{1} / \mathrm{FVC}$ could raise or lower the likelihood for lung emphysema in patients with COPD. The simple, parsimonious approach proposed by us might help if diagnostic resources regarding respiratory diseases are limited.

Trial registration ClinicalTrials.gov, Identifier: NCT01245933, registered 18 November 2010, https://clinicaltrials.gov/ct2/ show/record/NCT01245933.

Keywords: Emphysema, CT scan, Decision trees, Random forest, Adaboost, COPD phenotypes

\footnotetext{
*Correspondence: christina.kellerer@mri.tum.de

${ }^{1}$ School of Medicine, Institute of General Practice and Health Services

Research, Technische Universität München/Klinikum Rechts der Isar, Orleansstr. 47, 81667 Munich, Germany

Full list of author information is available at the end of the article
}

\section{Background}

Chronic obstructive pulmonary disease (COPD) is a common disorder with a high prevalence worldwide $[1,2]$. Lung emphysema is an important phenotype of COPD, and the differentiation between emphysema- and 
airway-phenotypes is increasingly relevant for the management of the disease. Currently, computed tomography of the chest $(\mathrm{CT})$ is the most precise method to detect, quantify and follow-up lung emphysema [3-5]. The differentiation between bronchitis and emphysema is important as emphysema shows functional characteristics different from those of chronic obstructive bronchitis [6-8], and patients with emphysema have partially different therapeutic options, such as lung volume reduction in case of severe hyperinflation [9-11], that are not relevant for predominant obstructive bronchitis. Current data show a protective effect of metformin on lung aging and thus on the development of emphysema, suggesting that in the future specific pharmacological therapeutic approaches might also become relevant in the treatment of emphysema [12]. Moreover, emphysema is associated with increased mortality risk and incidence of lung cancer $[13,14]$.

The clinical signs and symptoms of COPD can be quantified through questionnaires, such as the COPD Assessment Test (CAT) [1], modified Medical Research Council (mMRC) scale and St George's Respiratory Questionnaire (SGRQ) [1]. For CAT it has been demonstrated that single items confer information on emphysema [8]. SGRQ as a whole is too time-consuming for application in specialist's and non-specialist's practices, but has not been studied for the value of single items. The selected items could be combined with spirometry, often available in clinical practice. It would be of interest to compare this setting with the potential gain from functional measurements available only to the specialist, such as $\mathrm{CO}$ diffusing capacity, a method that is informative regarding lung emphysema $[7,15]$.

We analysed a subset $[8,16]$ of the COSYCONET (COPD and Systemic Consequences-Comorbidities Network) COPD cohort [17], comprising patients with prospective CT scans evaluated for the presence of emphysema. The aim was to identify a minimal subset of criteria that would increase or decrease the likelihood for emphysema to support clinical decision making for further diagnostic testing, such as CT. For this purpose, we used tree-based algorithms, either as single trees, or as statistical ensembles of trees. Via these approaches, we evaluated different sets of diagnostic criteria, ranging from single clinical symptoms to functional data available only to the pulmonary specialist.

\section{Methods}

\section{Study cohort}

Using the clinical and functional assessments in the multi-center COPD cohort COSYCONET [17], the present analysis was based on a subproject involving CT scans in inspiration and expiration under standardized conditions (for detailed information see Additional file 1). CT scans were performed around the time point of the third follow-up visit (visit 4), thus the functional and clinical data of this visit were used for analysis. At visit 4, 1427 of initially 2741 patients with COPD recruited at visit 1 still participated in COSYCONET, among these 1176 of spirometric GOLD grades 1-4. Of these 1427 patients, 518 participated in the CT substudy and had CT scans that could be evaluated qualitatively for the presence of either an airway-dominated or an emphysema-dominated phenotype. Among these patients, 436 showed GOLD grades 1 to 4 [1] at visit 4 and represented the present study population. CTs were assessed in 16 study centers, and their analysis was performed by experienced radiologists in the COSYCONET centre for image evaluation (University of Heidelberg); details can be found in the Additional file 1 . The binary emphysema score served as primary indicator of the COPD lung phenotype in all evaluations.

\section{Assessments}

Clinical history was assessed via standardized questionnaires [17], and clinical signs and symptoms via the instruments CAT [1], mMRC [1] and SGRQ [18]. Diagnosis of comorbidities were taken from the patients' reports of physician-based diagnoses. The lung function assessments evaluated comprised spirometry and diffusing capacity for carbon monoxide $(\mathrm{CO})$, which were performed according to SOPs following international guidelines and recommendations $[17,19]$. The parameters used in the present study were the forced expiratory volume in one second $\left(\mathrm{FEV}_{1}\right)$, forced expiratory volume (FVC), their ratio $\mathrm{FEV}_{1} / \mathrm{FVC}$, the transfer factor for $\mathrm{CO}$ (TLCO) and the transfer coefficient for CO (KCO). Predicted values were taken from the Global Lung Function Initiative (GLI) [20, 21].

\section{Statistical analysis}

Mean values and standard deviations (SD) were used to describe the distribution of quantitative data. Qualitative data is presented as absolute and relative frequencies. Hypothesis testing of group differences was performed by t-tests and Chi-squared tests, as appropriate. In order to obtain results that were best suited for potential application in clinical practice, we concentrated on single decision trees constructed with the exhaustive CHAID algorithm as implemented in SPSS [22]. Only binary branching was allowed to keep the trees simple, and all results given were based on tenfold cross validation and Bonferroni corrections to minimize errors. The analyses used all items of the questionnaires and optionally either spirometric, or spirometric and $\mathrm{CO}$ diffusing capacity data, with the aim to offer a wide panel of diverse 
information which was simple enough to be obtained in clinical practice. Thus, we selected the parameters offered to the algorithm under clinical and practical perspectives, but the final selection was based on statistical significance.

The trees' predictive performance and the relevance of the selected predictors were explored by comparison with the respective results of two commonly used and wellperforming reference methods. The first was the Random Forest approach, which is based on the construction of a random ensemble of decision trees [22, 23]. We used the standard settings of 500 trees and the number of variables chosen for splitting per node to be based on the square root of the number of variables. For this purpose, the package "randomForest" of the statistical software $\mathrm{R}$ (Version 4.0.2) was used [23, 24]. The second approach was the AdaBoost procedure that aims at the construction of a strong predictor by successive refinement of a set of weak predictors [24-26]. This method was realized using the packages "adabag" and "caret" from R [26]. To compare the methods we show the variables selected by these procedures in the rank order of importance according to the criterion of the mean decrease in accuracy (Random Forest) and the importance measure defined in the AdaBoost procedure, whereby the overall classification error refers to tenfold cross-validation in the case of AdaBoost and CHAID. All other computations were performed by SPSS Version 26 (IBM Corp., Armonk, NY, USA). Exploratory hypothesis testing was performed at two-sided significance levels of 0.05 .

\section{Results}

\section{Study cohort}

Of the 1427 patients participating in visit 4, 436 patients were eligible for analysis by having data on the presence of emphysema from the $\mathrm{CT}$ scoring and being categorized as COPD grades 1 to 4 (Table 1 ). The statistical comparison with patients not included in the CT analysis (Table 1) showed significant differences regarding $\mathrm{FEV}_{1}, \mathrm{FVC}$, in terms of \%predicted, moreover the distribution over GOLD grades and groups. All differences, however, were small, indicating that patients with $\mathrm{CT}$ showed slightly less severe COPD than patients without CT. Of the patients with CT, 185 showed an emphysemadominated, and 251 an airway-dominated phenotype of COPD.

\section{Tree-based prediction of emphysema}

In the decision and classification trees we focused on those combinations of values that showed either the greatest likelihood for emphysema dominance or the greatest likelihood for airway dominance (i.e. against emphysema) according to CT. Thus, we put the emphasis
Table 1 Patient characteristics

\begin{tabular}{lllr}
\hline Variables & Group without CT & Group with CT & p-value \\
\hline Sex (m/f) & $448 / 292$ & $271 / 165$ & 0.583 \\
Age (years) & $68.0 \pm 8.0$ & $66.2 \pm 8.2$ & $\mathrm{p}<0.001$ \\
BMl (kg/m $\left.{ }^{2}\right)$ & $26.7 \pm 5.2$ & $26.7 \pm 4.9$ & 0.898 \\
Packyears & $46.7 \pm 37.6$ & $46.8 \pm 36.8$ & 0.950 \\
Smok. never, ex-, act & $51 / 567 / 121$ & $27 / 319 / 90$ & 0.178 \\
FEV ${ }_{1} \%$ predicted & $51.1 \pm 18.6$ & $55.4 \pm 18.7$ & $\mathrm{p}<0.001$ \\
FVC \%predicted & $76.1 \pm 18.9$ & $82.6 \pm 20.3$ & $\mathrm{p}<0.001$ \\
FEV $/$ /FVC & $0.51 \pm 0.11$ & $0.51 \pm 0.11$ & 0.475 \\
TLCO \%predicted & $56.1 \pm 22.6$ & $59.6 \pm 22.7$ & 0.015 \\
KCO \%predicted & $64.3 \pm 25.6$ & $65.2 \pm 21.8$ & 0.531 \\
GOLD grades 1/2/3/4 & $54 / 310 / 283 / 93$ & $49 / 211 / 137 / 39$ & 0.003 \\
GOLD groups ABCD & $237 / 163 / 113 / 222$ & $170 / 86 / 80 / 99$ & 0.014 \\
\hline
\end{tabular}

Comparison of the subgroup with CT scans (study population) and the patients without CT scans from COSYCONET that were participants at the time of the CT scans. Statistical comparisons were performed with either t-tests or Chi-square contingency tables, as appropriate

on the combinations of values yielding the best predictions, neglecting all other combinations that showed only slight changes in the likelihood of emphysema. Decision trees allow to identify not only values that are informative but also values that are not informative [22]. This was done for the set of all questionnaire items (CAT, SGRQ, $\mathrm{mMRC}$ ), moreover their combination with spirometric parameters including $\mathrm{FEV}_{1} / \mathrm{FVC}$.

The respective trees are shown in Figs. 1 and 2. It can be seen that odds ratios for emphysema ranged above 4 and that the combination of CAT item 1 (cough), mMRC and $\mathrm{FEV}_{1} / \mathrm{FVC}$ yielded an odds ratio of more than 7 , while odds ratios for the absence of emphysema tended to be lower. Importantly, the trees demonstrate that some combinations of individual values, i.e. combinations of binary partitions and respective definitions of patient subgroups, were highly informative compared to baseline but other combinations not, irrespective of the fact that the partitions at each node were statistically significant. For practical purposes, Fig. 4 summarizes the results of the decision trees in a single diagram, whereby we selected the nodes showing the maximum odds ratios for the emphysema- or airway-dominant phenotype.

To assess the prediction of emphysema including $\mathrm{CO}$ diffusing capacity, we repeated the sequence of analyses with the data from clinical history/questionnaires and spirometry but additionally diffusing capacity. The respective tree is shown in Fig. 3 and the results are included in Fig. 4. KCO \%predicted was the primary decision parameter, followed by $\mathrm{FEV}_{1} / \mathrm{FVC}$, while CAT item 4 (breathlessness) and mMRC were additionally relevant. It should be noted that high values of $\mathrm{KCO}$ and $\mathrm{FEV}_{1} /$ FVC without the CAT item 4 already resulted in a high 
Airway-dominant type

Emphysema-dominant type

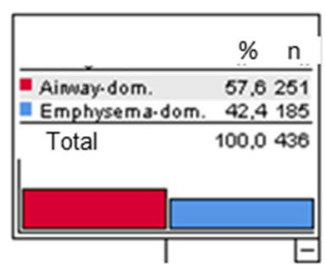

SGRQ item 8, chest condition

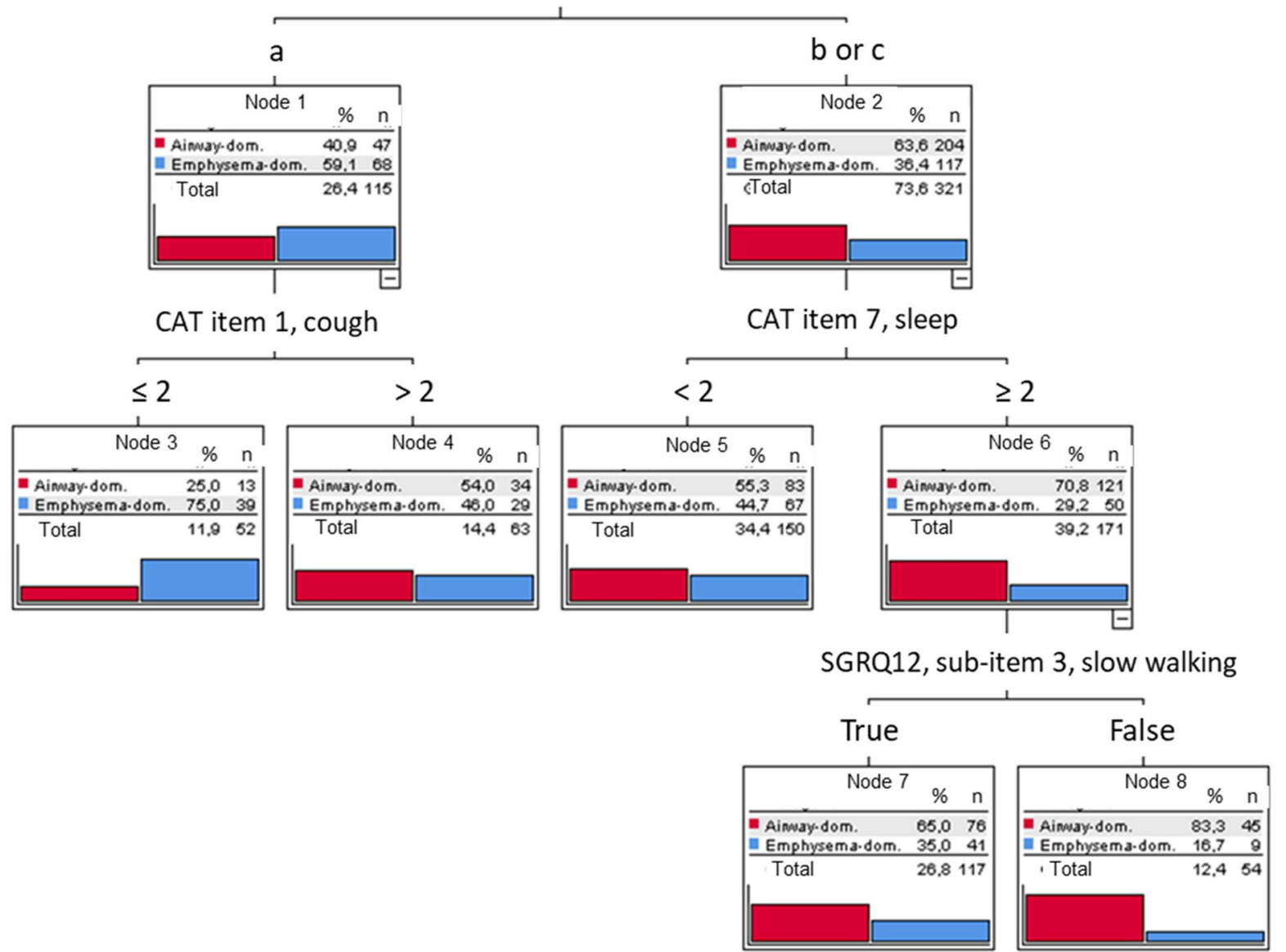

Fig. 1 Decision tree derived from the inclusion of CAT, SGRQ and mMRC. Only CAT items 1 and 7 as well as SGRQ items 8 and SGRQ 12 sub-item 3 were selected as significant predictors. Item 8 of the SGRQ is the question „How would you describe your chest condition?" with following answer options a) Causes me a lot of problems or is the most important problem I have, b) Causes me a few problems and c) Causes no problem. Please note the large differences in the distribution of diagnoses, whereby the final nodes 4, 5 and 7 were scarcely informative compared to the prior values (comprising 327 patients). In contrast, nodes 3 and 8 were informative (comprising 106 patients). The odds ratio for emphysema corresponding to node 3 was 4.06

odds ratio for the airway-dominant type, as indicated in node 6 in Fig. 3.

\section{Comparison of decision trees with machine learning results}

The machine learning methods Random Forest and AdaBoost are often used to improve the predictive performance of classification trees. We used these procedures to assess whether the overall accuracy was similar to that of the single trees. If it would be markedly higher, this would point to a classification problem of higher complexity that cannot be adequately solved by a classification tree. The results are shown in the Additional file 1: Table S1, indicating that the sets of important variables contained those selected in the single decision trees. Moreover, the overall errors were similar in both the machine learning approaches 
Airway-dominant type

Emphysema-dominant type

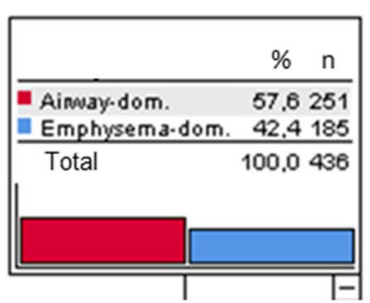

\section{$\mathrm{FEV}_{1} / \mathrm{FVC}$}

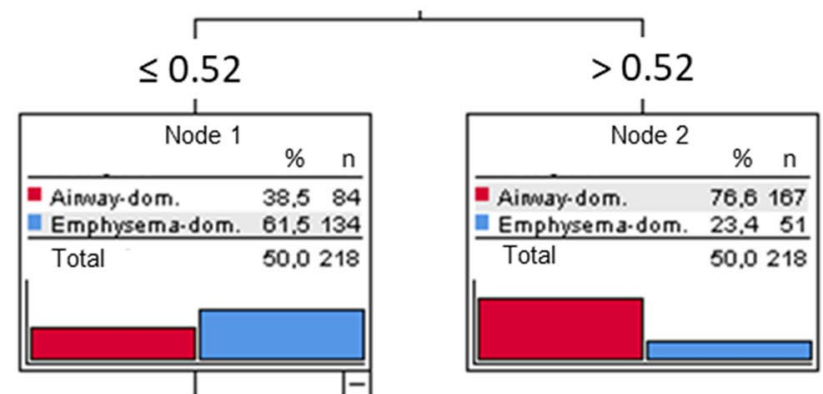

CAT item 1, cough
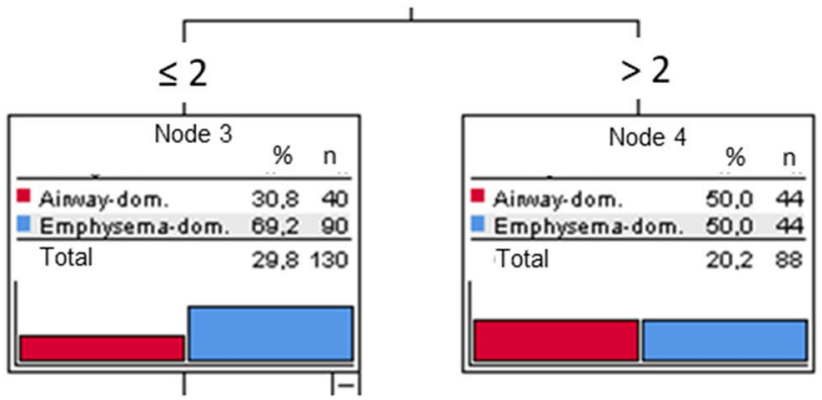

$\mathrm{mMRC}$

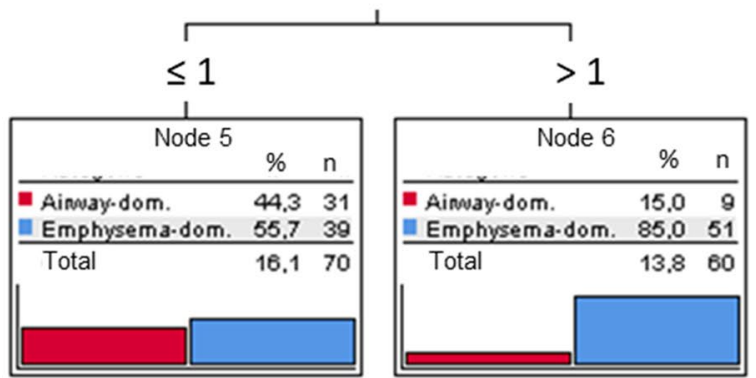

Fig. 2 Decision tree derived from the inclusion of CAT, SGRQ, mMRC and FEV $1 / F V C$. Only item 1 from CAT, mMRC and FEV $/$ /FVC were selected as significant predictors. Please note the large differences in the distribution of diagnoses; nodes 2 and 6 were maximally informative ( $n=278$ patients), nodes 4 and 5 less informative ( $n=158$ patients). The odds ratio for emphysema corresponding to node 6 was 7.69

and the single decision trees. It should be noted that the overall error comprises the combinations of values that were not informative as illustrated in Figs. 1, 2, 3 , thereby overestimating the error obtained when 
Airway-dominant type Emphysema-dominant type

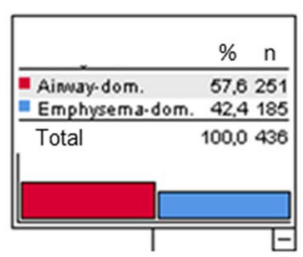

KCO \%predicted

\section{$\leq 63.2$}

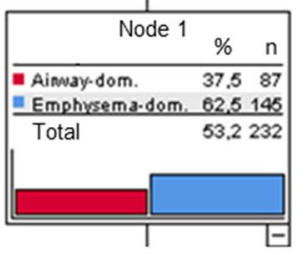

$\mathrm{FEV}_{1} / \mathrm{FVC}$

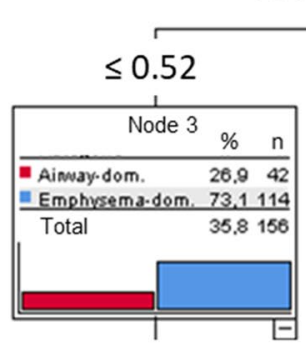

mMRC

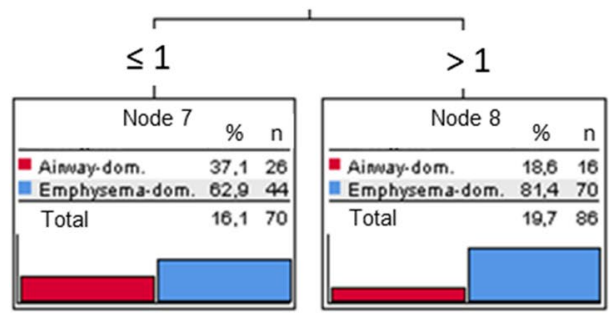

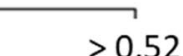
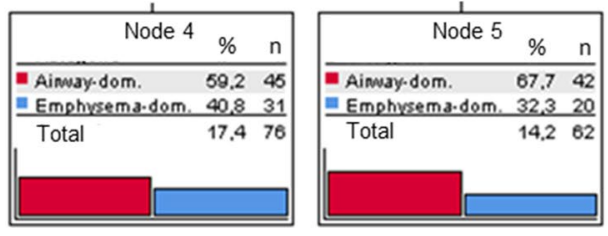

$>63.2$

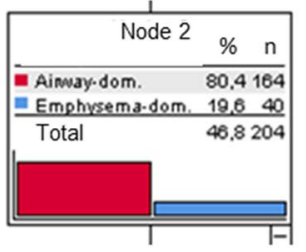

$\mathrm{FEV}_{1} / \mathrm{FVC}$
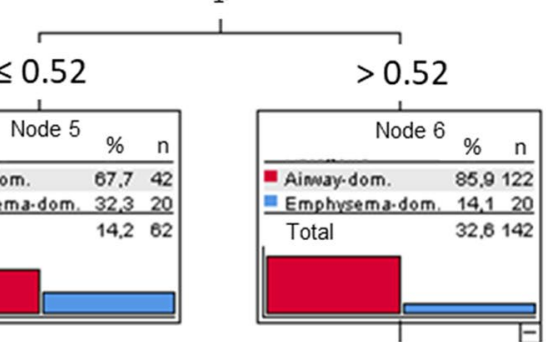

CAT item 4, breathlessness

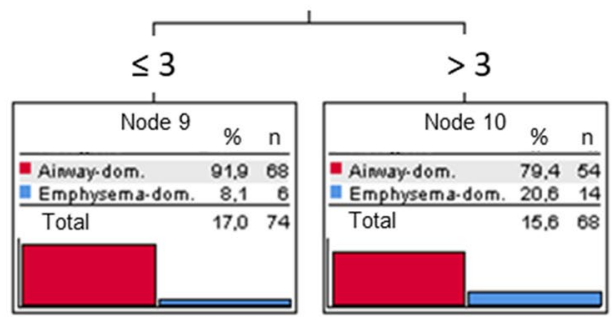

Fig. 3 Decision tree derived from the inclusion of CAT, SGRQ, mMRC, spirometric, and CO diffusing capacity parameters. Only item 4 from CAT, $\mathrm{mMRC}, \mathrm{KCO} \%$ predicted and $\mathrm{FEV}_{1} / \mathrm{FVC}$ were selected as significant predictors. Please note the large differences in the distribution of diagnoses; nodes 8 and 9 were maximally informative ( $n=160$ patients), nodes 4, 5, 7 and 10 less informative ( $n=276$ patients). The odds ratio for emphysema corresponding to node 8 was 5.51

restricting to the cases with high odds ratios shown in Fig. 4.

\section{Discussion}

The present analysis had the aim to identify criteria by which physicians with limited technical resources regarding respiratory diseases can improve the diagnosis of emphysema in COPD patients. This could be achieved by a small set of questions combined with spirometry, whereby the responses and values indicated either a markedly elevated or a lowered likelihood for the emphysema-dominated phenotype. This might strengthen the rationale for performing quantitative assessment of the lung with CT. We evaluated all single items of the CAT and SGRQ, as well as the mMRC, supplemented by spirometric data, and for comparison also $\mathrm{CO}$ diffusing capacity. In the decision trees, three items of the CAT, two items of the SGRQ, as well as the mMRC were informative. Among spirometric parameters, $\mathrm{FEV}_{1} / \mathrm{FVC}$ was most informative, and among the parameters of $\mathrm{CO}$ diffusing capacity, the transfer coefficient (KCO) expressed as \%predicted. This was evident in the decision trees from those combinations of values that maximally raised the likelihood for an emphysema- or airway-dominated 


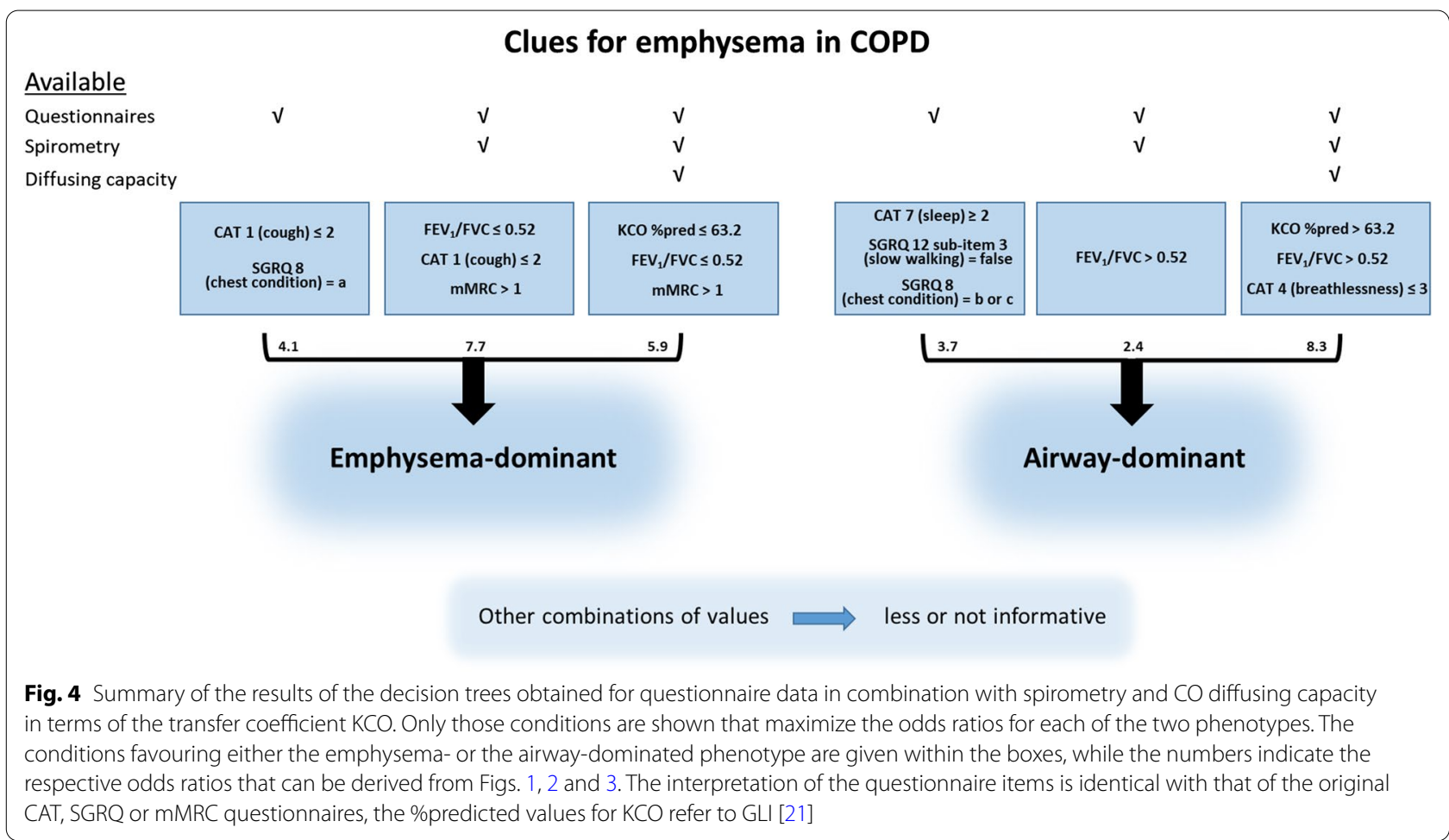

phenotype. In combination with $\mathrm{FEV}_{1} / \mathrm{FVC}$, odds ratios ranged up to values of more than 7 . Using diffusing capacity, the odds ratio for the airway-dominated phenotype reached a similar value. Our results demonstrate that a few anamnestic questions plus spirometric data can provide significant evidence on the presence of lung emphysema which subsequently can be substantiated by ordering a CT scan.

The most precise diagnosis and quantification of lung emphysema is achieved by chest CT but due to limited availability and cost restrictions, this is not yet clinical routine. As the identification of patients with emphysema is relevant for therapeutic decisions and interventions, all information supporting a well-founded referral for quantitative $\mathrm{CT}$ is helpful. If selected questionnaire items are associated with emphysema, as demonstrated for the CAT [8], this is particularly useful, since long questionnaires are difficult to implement in the busy routine of a family physician's practice. With regard to CAT and $\mathrm{mMRC}$, the present results were consistent with the previous observations of correlation patterns [8] while for the SGRQ single item data are not available. The single item approach was essential in finding concise algorithms that might be particularly suited for a family physician's daily routine with limited diagnostic resources regarding respiratory diseases (see Fig. 4).

We focused on patients with either a high or a low likelihood of emphysema. Only in these cases, we expected that algorithms based on few simple questions can be efficient. Conversely, the decision trees indicated, that in about half or more of the cases the changes in likelihood were low. Such patients, in whom an informed decision well supported with the available data, would need further diagnostic evaluation, i.e. referral to a specialist, and we consider their identification an advantage. When computing overall sensitivity for emphysema by averaging over all conditions, values were $21.1 \%, 72.4 \%$, and $61.6 \%$ for questionnaire items alone, questionnaires combined with spirometry, and the combination of questionnaires, spirometry and $\mathrm{KCO}$, respectively. Conversely, the sensitivity for the airway-dominated type was $94.8 \%$, $66.5 \%$, and $83.3 \%$, respectively.

To estimate the maximum accuracy, we evaluated the gain by adding data of $\mathrm{CO}$ diffusion capacity as typically obtained in a pulmonologist's practice. As expected, $\mathrm{CO}$ diffusing capacity conferred the primary information on the presence of emphysema, in line with previous results delineating the cumulative value of spirometry, diffusing capacity and bodyplethysmography [7]. From Fig. 4 and the comparison of the decision trees shown in Figs. 2 and 3 , it appeared that the major benefit from KCO referred to the recognition of the airway-dominated phenotype.

Decision trees are susceptible to overfitting which we tried to reduce by using tenfold cross validation. In order to check the results, we additionally employed machine learning methods that rely on ensembles of trees or a 
sequence of consecutively refined trees. For this purpose, we performed a sensitivity analysis, using the Random Forest and the AdaBoost approach. This related but different approach confirmed that the questionnaire items and functional parameters revealed as important comprised the variables identified in the single decision trees. We did not use this as primary analysis as it does not result in directly comprehensible trees.

Figure 4 summarizes the clues for an emphysema- versus airway-dominated phenotype in a comprehensive and easily applicable form. For example, a low symptom burden from cough (CAT item $1 \leq 2)$ combined with shortness of breath at common exercise levels $(m M R C>1)$ and an impaired ratio $\mathrm{FEV}_{1} / \mathrm{FVC}(\leq 0.52)$ raised the likelihood of emphysema by a factor of about 7.7. This underlines that easily obtained information can be sufficient for an informed decision, for example regarding the order of a CT-scan, independent from other diagnostic intentions, for example regarding lung cancer. It might be surprising that smoking history did not play a role in the decision trees, but this was probably due the fact that we included only patients with an established diagnosis of COPD. Moreover, when $\mathrm{FEV}_{1} / \mathrm{FVC}$ was included, the values of $\mathrm{FEV}_{1}$ \%predicted and correspondingly GOLD grades did not provide additional significant information, at least with the maximum tree depths of 3 which we fixed in order to keep the results robust and interpretable. For clinical practice, the COPD phenotype is of interest. For example, patients with advanced emphysema may benefit from lung volume reduction procedures in case of severe hyperinflation [11], and recent data described a relationship between cigarette smokeinduced oxidative stress and inflammation, leading to enhanced lung aging, apoptosis and emphysema [12]. The data also provided evidence for protective effects of metformin on the progression of emphysema [12]. These findings point towards potential future treatment options for emphysema and emphasize the need to determine the dominant phenotype.

\section{Limitations}

The analysis was based on a subset of the COSYCONET cohort, and there were slight differences between patients having a CT scan versus those having no scan. In principle, differences between this cohort and typical primary care populations are possible but there are no sufficient data on this. At least, the percentage of emphysemadominated type (42.4\%) was similar to those observed in other large COPD studies [27, 28]. There is no evidence that possible differences between populations affected the validity of the decision trees, especially the identification of informative vs non-informative nodes. Therefore, further studies with typical populations of clinical practices and different a priori likelihood for COPD and emphysema would be useful. It should be kept in mind that our analysis refers to patients who have already the diagnosis of COPD, therefore symptoms such as slow walking and sleep disturbance are to be interpreted on this background. Without prior diagnosis of COPD, these symptoms will be less indicative of emphysema; our study aimed at providing simple and easy diagnostic help for specific conditions not in general. The limited size of the dataset also limited the maximum depth of the decision trees, as we followed the standard requirement of a minimum number of 50 patients in each node. This was no disadvantage in terms of usability, as trees with many levels would be less applicable than simple trees. In addition, we consider the risk of overfitting as minor, as we performed cross-validation and the variables identified in the single decision trees were among the variables identified in the machine learning algorithms. Another limitation is that our findings were based on a secondary analysis, thus the findings need to be validated in a confirmatory study, ideally in a family physicians' setting with limited diagnostic resources regarding respiratory diseases. Due to the selection of patients participating in a clinical study such as COSYCONET, it cannot be anticipated, how robust the suggested approach will work in clinical settings involving a larger spectrum of differential diagnoses that have an impact on symptoms, such as cardiac diseases. It therefore might be a next step to perform a similar analysis in family physicians' cohorts.

\section{Conclusions}

Emphysema is an important phenotype of COPD and commonly diagnosed via chest CT scans involving additional costs and radiation exposure. Provided that a diagnosis of COPD has been established, the use of few single items of COPD questionnaires in combination with $\mathrm{FEV}_{1} / \mathrm{FVC}$ significantly raised or lowered the likelihood of an emphysema- versus airway-dominated COPD phenotype in a large proportion of patients. The simple, easy to apply criteria proposed by us might be useful in clinical practice for the decision of ordering a CT scan, particularly in non-pulmonary specialist settings, such as family physicians. The second result was that patients with answers and $\mathrm{FEV}_{1} / \mathrm{FVC}$ values that did not markedly change the likelihood could also be identified, which might be helpful in the decision to refer them to pulmonary specialists.

\footnotetext{
Abbreviations

CAT: COPD Assessment Test; COPD: Chronic obstructive pulmonary disease; CT: Computed tomography; FEV1: Forced expiratory volume in one second; FVC: Forced expiratory volume; mMRC: Modified Medical Research Council scale; SD: Standard deviation; SGRQ: St George's Respiratory Questionnaire.
} 


\section{Supplementary Information}

The online version contains supplementary material available at https://doi. org/10.1186/s12931-021-01837-2.

Additional file 1: Table S1. Results of the Random Forest and AdaBoost procedures for different sets of variables included. The table shows the variables in the rank order of importance according to the criterion of the mean decrease in accuracy (Random Forest) and the importance measure defined in the AdaBoost procedure. The overall classification error refers to 10 -fold cross-validation in the case of AdaBoost and CHAID. SGRQ4 "I have attacks of wheezing", SGRQ5 "How many attacks of chest trouble did you have during the last year?", SGRQ8, "How would you describe your chest condition?", SGRQ11 sub-item 5"I have become frail or an invalid because of my chest", SGRQ12 sub-item 1 "I take a long time to get washed or dressed", SGRQ12 sub-item 3"I walk slower than other people, or I stop for rests", SGRQ12 sub-item 4 "Jobs such as housework take a long time, or I have to stop for rests", SGRQ13 sub-item 1 "I cannot play sports or games", SGRQ14"How does your chest trouble affect you? Table S2. Technical details of the CT assessment. The acquisition protocol is given in the upper part and details on the scanner models in the lower part of the table. *Vendor-specific generic names for Siemens/GE/Philips. Table S3. Items from the "St. George's Respiratory Questionnaire" (SGRQ) that turned out to be informative in the single decision trees. Table S4. Items from the COPD Assessment test (CAT). Table S5. Modified Medical Research Council (mMRC) scale. This self-rating questionnaire is used to measure the degree of disability that breathlessness poses on day-to-day activities on a scale from 0 to 4 .

\section{Acknowledgements}

We would like to thank all patients for their kind participation as well as the COSYCONET Study-Group and the participating study nurses for their efforts. COSYCONET Study-Group: Andreas, Stefan (Lungenfachklinik, Immenhausen); Bals, Robert (Universitätsklinikum des Saarlandes); Behr, Jürgen and Kahnert, Kathrin (Klinikum der Ludwig-Maximilians-Universität München); Bewig, Burkhard and Bahmer, Thomas (Universitätsklinikum Schleswig Holstein); Buhl, Roland (Universitätsmedizin der Johannes-Gutenberg-Universität Mainz); Ewert, Ralf and Stubbe, Beate (Universitätsmedizin Greifswald); Ficker, Joachim H. (Klinikum Nürnberg, Paracelsus Medizinische Privatuniversität Nürnberg); Gogol, Manfred (Institut für Gerontologie, Universität Heidelberg); Grohé, Christian (Ev. Lungenklinik Berlin); Hauck, Rainer (Kliniken Südostbayern AG, Kreisklinik Bad Reichenhall); Held, Matthias and Jany, Berthold (Klinikum Würzburg Mitte gGmbH, Standort Missioklinik); Henke, Markus (Asklepios Fachkliniken München-Gauting); Herth, Felix (Thoraxklinik Heidelberg gGmbH); Höffken, Gerd (Fachkrankenhaus Coswig GmbH); Katus, Hugo A. (Universitätsklinikum Heidelberg); Kirsten, Anne-Marie and Watz, Henrik (Pneumologisches Forschungsinstitut an der Lungenclinic Grosshansdorf GmbH); Koczulla, Rembert and Kenn, Klaus (Schön Klinik Berchtesgadener Land); Kronsbein, Juliane (Berufsgenossenschaftliches Universitätsklinikum Bergmannsheil, Bochum); Kropf-Sanchen, Cornelia (Universitätsklinikum UIm); Lange, Christoph and Zabel, Peter (Forschungszentrum Borstel); Pfeifer, Michael (Klinik Donaustauf); Randerath, Winfried J. (Wissenschaftliches Institut Bethanien e. V., Solingen); Seeger, Werner (Justus-Liebig-Universität Gießen); Studnicka, Michael (Uniklinikum Salzburg); Taube, Christian and Teschler, Helmut (Ruhrlandklinik gGmbH Essen); Timmermann, Hartmut (Hamburger Institut für Therapieforschung GmbH); Virchow, J. Christian (Universitätsklinikum Rostock); Vogelmeier, Claus (Universitätsklinikum Gießen und Marburg GmbH, Standort Marburg); Wagner, Ulrich (Klinik Löwenstein gGmbH); Welte, Tobias (Medizinische Hochschule Hannover); Wirtz, Hubert (Universitätsklinikum Leipzig). Names of participating study nurses: Lehnert, Doris, Evangelische Lungenklinik Berlin; Struck, Birte, Bergmannsheil Berufsgenossenschaftliches Universitätsklinikum Bochum; Krabbe, Lenka, Medizinische-Klinik Borstel; Arikan, Barbara, Tobias, Julia, Klinik Donaustauf; Spangel, Gina, Teng, Julia, Ruhrlandklinik gGmbH Essen, Speth, Kornelia, Universitätsklinikum Gießen; Pieper, Jeanette, Universitätsmedizin Greifswald; Gleiniger, Margret, Markworth, Britta, Hinz, Zaklina, Hundack-Winter, Petra, Pneumologisches Forschungsinstitut Großhansdorf; Burmann, Ellen, Hamburger Institut für Therapieforschung Hamburg; Wons, Katrin, Wagner, Sylvia, Medizinische Hochschule Hannover; Rieber, Ulrike, Schaufler, Beate, Thoraxklinik am Universitätsklinikum Heidelberg; Seibert,
Martina, Universitätsklinikum des Saarlandes, Homburg/Saar; Schwedler, Katrin, Lungenfachklinik Immenhausen; Michalewski, Sabine, Rohweder, Sonja, Universitätsklinikum Schleswig-Holstein, Campus Kiel; Berger, Patricia, Universitätsklinikum Leipzig; Schottel, Diana, Krankenhaus Lindenbrunn, Coppenbrügge; Klöser, Manuel, Universitätsmedizin der Johannes GutenbergUniversität Mainz; Janke, Vivien, Universitätsklinikum Marburg; Untsch, Rosalie, Asklepios Fachkliniken, München-Gauting; Graf, Jana, Graf, Veronika, Klinikum der Universität München; Reichel, Anita, Klinikum Nürnberg; Weiß, Gertraud, Traugott, Erich, Ziss, Barbara, Schön Klinik Berchtesgadener Land; Kietzmann, Ilona, Wissenschaftliches Institut Bethanien für Pneumologie e. V, Solingen; Schrade-IIImann, Michaela, Polte, Beate, Universitätsklinikum-Ulm; Böckmann, Cornelia, Hübner, Gudrun, Sterk, Lena, Wirz, Anne, Klinikum Würzburg Mitte gGmbH, Standort Missioklinik, Würzburg.

\section{Authors' contributions}

CK was involved in the conception of the study, analyzing and interpreting the data, statistical analysis, conceptualizing and drafting of the manuscript, approved the final submitted version, and agreed to be accountable for all aspects of the work. RAJ was involved in the conception of the study, analyzing and interpreting the data, statistical analysis, conceptualizing and drafting of the manuscript, approved the final submitted version, and agreed to be accountable for all aspects of the work. AS was involved in the interpretation of the data from this analysis, took part in the discussion and critical revision of this manuscript, approved the final submitted version, and agreed to be accountable for all aspects of the work. PA was involved in the interpretation of the data from this analysis, took part in the discussion and critical revision of this manuscript, approved the final submitted version, and agreed to be accountable for all aspects of the work. H-UK was involved in the interpretation of the data from this analysis and in interpretation of the CT scans, took part in the discussion and critical revision of this manuscript, approved the final submitted version, and agreed to be accountable for all aspects of the work. BJ was involved in the interpretation of the data from this analysis and in interpretation of the CT scans, took part in the discussion and critical revision of this manuscript, approved the final submitted version, and agreed to be accountable for all aspects of the work. JB was involved in the interpretation of the data from this analysis and in interpretation of the CT scans, took part in the discussion and critical revision of this manuscript, approved the final submitted version, and agreed to be accountable for all aspects of the work. RB was involved in the interpretation of the data from this analysis and drafting of the manuscript, approved the final submitted version, and agreed to be accountable for all aspects of the work. HW was involved in the interpretation of the data from this analysis and drafting of the manuscript, approved the final submitted version, and agreed to be accountable for all aspects of the work. JB was involved in the interpretation of the data from this analysis, took part in the discussion and critical revision of this manuscript, approved the final submitted version, and agreed to be accountable for all aspects of the work. DK-G was involved in the interpretation of the data from this analysis, took part in the discussion and critical revision of this manuscript, approved the final submitted version, and agreed to be accountable for all aspects of the work. JL was involved in the interpretation of the data from this analysis, took part in the discussion and critical revision of this manuscript, approved the final submitted version, and agreed to be accountable for all aspects of the work. AH was involved in the statistical analysis and critical revision of the manuscript, approved the final submitted version, and agreed to be accountable for all aspects of the work. HM was involved in the interpretation of the data from this analysis, took part in the discussion and critical revision of this manuscript, approved the final submitted version, and agreed to be accountable for all aspects of the work. FT was involved in the interpretation of the data from this analysis, took part in the discussion and critical revision of this manuscript, approved the final submitted version, and agreed to be accountable for all aspects of the work. TW contributed to the overall design of COSYCONET, to the interpretation of the data from this analysis, to the development and critical revision of the manuscript, approved the final submitted version, and agreed to be accountable for all aspects of the work. CV contributed to the overall design of COSYCONET, to the interpretation of the data from this analysis, to the development and critical revision of the manuscript, approved the final submitted version, and agreed to be accountable for all aspects of the work. KK was involved in the conception of the study, statistical analysis and interpretation of the data, conceptualizing and drafting of the manuscript, approved the final submitted version, and agreed 
to be accountable for all aspects of the work. All authors read and approved the final manuscript.

\section{Funding}

Open Access funding enabled and organized by Projekt DEAL. This work is supported by the German Centre for Lung Research (DZL), Grant number 82DZLI05A2 (COSYCONET), the BMBF, Grant number $01 \mathrm{Gl} 0881$ and is furthermore supported by unrestricted grants from AstraZeneca $\mathrm{GmbH}$, Chiesi $\mathrm{GmbH}$, GlaxoSmithKline GmbH\&Co. KG, Grifols Deutschland GmbH, Novartis Deutschland $\mathrm{GmbH}$. The funding body had no involvement in the design of the study, or the collection, analysis or interpretation of the data.

\section{Availability of data and materials}

The basic data are part of the German COPD cohort COSYCONET (www.ascon et.net/) and available upon request. There is a detailed procedure for this on the website of this network. Specifically, the data can be obtained by submission of a proposal that is evaluated by the steering committee. All results to which the manuscript refers, are documented appropriately in the text, figures or tables.

\section{Declarations}

\section{Ethics approval and consent to participate}

All assessments were approved by the central (Marburg (Ethikkommission FB Medizin Marburg) and local (Bad Reichenhall (Ethikkommission bayerische Landesärztekammer); Berlin (Ethikkommission Ärztekammer Berlin); Bochum (Ethikkommission Medizinische Fakultät der RUB); Borstel (Ethikkommission Universität Lübeck); Coswig (Ethikkommission TU Dresden); Donaustauf (Ethikkommission Universitätsklinikum Regensburg); Essen (Ethikkommission Medizinische Fakultät Duisburg-Essen); Gießen (Ethikkommission Fachbereich Medizin); Greifswald (Ethikkommission Universitätsmedizin Greifswald); Großhansdorf (Ethikkommission Ärztekammer Schleswig-Holstein); Hamburg (Ethikkommission Ärztekammer Hamburg); MHH Hannover / Coppenbrügge (MHH Ethikkommission); Heidelberg Thorax/Uniklinik (Ethikkommission Universität Heidelberg); Homburg (Ethikkommission Saarbrücken); Immenhausen (Ethikkommission Landesärztekammer Hessen); Kiel (Ethikkommission Christian-Albrechts-Universität zu Kiel); Leipzig (Ethikkommission Universität Leipzig); Löwenstein (Ethikkommission Landesärztekammer Baden-Württemberg); Mainz (Ethikkommission Landesärztekammer Rheinland-Pfalz); München LMU/Gauting (Ethikkommission Klinikum Universität München); Nürnberg (Ethikkommission Friedrich-Alexander-Universität Erlangen Nürnberg); Rostock (Ethikkommission Universität Rostock); Berchtesgadener Land (Ethikkommission Land Salzburg); Schmallenberg (Ethikkommission Ärztekammer Westfalen-Lippe); Solingen (Ethikkommission Universität WittenHerdecke); Ulm (Ethikkommission Universität UIm); Würzburg (Ethikkommission Universität Würzburg) Ethical Committees, and written informed consent was obtained from all patients. The study was based on 2741 patients recruited within the COSYCONET framework (ClinicalTrials.gov, Identifier: NCT01245933, registered 18 November 2010, https://clinicaltrials.gov/ct2/ show/record/NCT01245933). For further information see: [17].

\section{Consent for publication}

Within the ethical approval, the participants of the study gave their consent to publish the data collected during the study period.

\section{Competing interests}

C. Kellerer, R. A. Jörres, A. Schneider, B. Jobst, J. Biederer, H. Watz, J. Behr, D. Kauffmann-Guerrero, A. Hapfelmeier, H. Magnussen, F. C. Trudzinski, and K. Kahnert have nothing to disclose with regard to this study.P. Alter reports grants from German Federal Ministry of Education and Research (BMBF) Competence Network Asthma and COPD (ASCONET), grants from AstraZeneca $\mathrm{GmbH}$, grants and non-financial support from Bayer Schering Pharma AG, grants, personal fees and non-financial support from Boehringer Ingelheim Pharma GmbH \& Co. KG, grants and non-financial support from Chiesi $\mathrm{GmbH}$, grants from GlaxoSmithKline, grants from Grifols Deutschland $\mathrm{GmbH}$, grants from MSD Sharp \& Dohme GmbH, grants and personal fees from Mundipharma GmbH, grants, personal fees and non-financial support from Novartis Deutschland $\mathrm{GmbH}$, grants from Pfizer Pharma $\mathrm{GmbH}$, grants from Takeda Pharma Vertrieb GmbH \& Co. KG, outside the submitted work.H.-U. Kauczor reports non-financial support from Bayer, non-financial support from Siemens, during the conduct of the study; grants from Siemens, grants and personal fees from Philips, personal fees from Boehringer Ingelheim, personal fees from Merck Sharp Dohme, personal fees from Astra Zeneca, outside the submitted work.R. Bals reports grants and personal fees from AstraZeneca, grants and personal fees from Boehringer Ingelheim, personal fees from GlaxoSmithKline, personal fees from Grifols, grants and personal fees from Novartis, personal fees from CSL Behring, grants from German Federal Ministry of Education and Research (BMBF) Competence Network Asthma and COPD (ASCONET), grants from Sander Stiftung, grants from Schwiete Stiftung, grants from Krebshilfe, grants from Mukoviszidose eV, outside the submitted work.J. Lutter reports grants from German Federal Ministry of Education and Research (BMBF) with grant number 01Gl0882, during the conduct of the study.T. Welte reports grants from German Ministry of Education and Research (BMBF), during the conduct of the study; personal fees from AstraZeneca, GSK, Novartis, CSL Behring, outside the submitted work.C. Vogelmeier reports grants and personal fees from AstraZeneca, grants and personal fees from Boehringer Ingelheim, personal fees from CSL Behring, personal fees from Chiesi, grants and personal fees from GlaxoSmithKline, grants and personal fees from Grifols, personal fees from Menarini, grants and personal fees from Novartis, personal fees from Nuvaira, personal fees from MedUpdate, outside the submitted work.

\section{Author details}

${ }^{1}$ School of Medicine, Institute of General Practice and Health Services Research, Technische Universität München/Klinikum Rechts der Isar, Orleansstr. 47, 81667 Munich, Germany. ${ }^{2}$ Institute and Outpatient Clinic for Occupational, Social and Environmental Medicine, Comprehensive Pneumology Center Munich (CPC-M), Ludwig-Maximilians-Universität München, Ziemssenstr. 1, 80336 Munich, Germany. ${ }^{3}$ Department of Medicine, Pulmonary and Critical Care Medicine, University Medical Center Giessen and Marburg, PhilippsUniversity Marburg, German Center for Lung Research (DZL), Baldingerstrasse, 35043 Marburg, Germany. ${ }^{4}$ Department of Diagnostic and Interventional Radiology, University Hospital of Heidelberg, Heidelberg, Germany. ${ }^{5}$ Translational Lung Research Centre Heidelberg (TLRC), Member of the German Center for Lung Research, Heidelberg, Germany. ${ }^{6}$ Faculty of Medicine, University of Latvia, Raina bulvaris 19, Riga 1586, Latvia. ${ }^{7}$ Faculty of Medicine, ChristianAlbrechts-Universität Zu Kiel, 24098 Kiel, Germany. ${ }^{8}$ Department of Internal Medicine V - Pulmonology, Allergology, Respiratory Intensive Care Medicine, Saarland University Hospital, Kirrberger Straße 1, 66424 Homburg, Germany. ${ }^{9}$ Airway Research Center North (ARCN), German Center for Lung Research (DZL), Pulmonary Research Institute at LungenClinic Grosshansdorf, Woehrendamm 80, 22927 Grosshansdorf, Germany. ${ }^{10}$ Department of Internal Medicine V, University of Munich (LMU), Comprehensive Pneumology Center, German Center for Lung Research, Ziemssenstr. 1, 80336 Munich, Germany. ${ }^{11}$ Comprehensive Pneumology Center Munich (CPC-M), German Center for Lung Research (DZL), Institute of Epidemiology, Helmholtz Zentrum München $(\mathrm{GmbH})$ - German Research Center for Environmental Health, 85764 Neuherberg, Germany. ${ }^{12}$ Translational Lung Research Centre Heidelberg (TLRC), Member of the German Center for Lung Research, Thoraxklinik-Heidelberg gGmbH, Röntgenstraße 1, 69126 Heidelberg, Germany. ${ }^{13}$ Department of Pneumology, Hannover Medical School, Carl-Neuberg-Str. 1, 30625 Hannover, Germany.

Received: 2 February 2021 Accepted: 1 September 2021 Published online: 09 September 2021

\section{References}

1. Vogelmeier CF, et al. Global strategy for the diagnosis, management, and prevention of chronic obstructive lung disease 2017 report. GOLD executive summary. Am J Resp Crit Care Med. 2017;195(5):557-82.

2. Afonso AS, et al. COPD in the general population: prevalence, incidence and survival. Respir Med. 2011;105(12):1872-84.

3. Lutchmedial SM, et al. How common is airflow limitation in patients with emphysema on CT scan of the chest? Chest. 2015;148(1):176-84.

4. Newell J, Hogg J, Snider G. Report of a workshop: quantitative computed tomography scanning in longitudinal studies of emphysema. Eur Respir J. 2004;23(5):769-75. 
5. Barr CC, et al. A combined pulmonary-radiology workshop for visual evaluation of COPD: study design, chest CT findings and concordance with quantitative evaluation. COPD J Chronic Obstructive Pulm Dis. 2012;9(2):151-9.

6. Kitaguchi Y, et al. Characteristics of COPD phenotypes classified according to the findings of HRCT. Respir Med. 2006;100(10):1742-52.

7. Kahnert K, et al. Relationship of spirometric, body plethysmographic, and diffusing capacity parameters to emphysema scores derived from CT scans. Chron Respir Dis. 2018:16:1479972318775423.

8. von Siemens SM, et al. CAT score single item analysis in patients with COPD: results from COSYCONET. Respir Med. 2019;159:105810.

9. Subramanian DR, et al. Emphysema-and airway-dominant COPD phenotypes defined by standardised quantitative computed tomography. Eur Respir J. 2016;48(1):92-103.

10. Johannessen A, et al. Mortality by level of emphysema and airway wall thickness. Am J Respir Crit Care Med. 2013;187(6):602-8.

11. Herth FJ, et al. Endoscopic lung volume reduction: an expert panel recommendation-update 2017. Respiration. 2017:94(4):380-8.

12. Polverino F, et al. Metformin: experimental and clinical evidence for a potential role in emphysema treatment. Am J Resp Crit Care Med. 2021 https://doi.org/10.1164/rccm.202012-45100C.

13. Lynch DA, et al. CT-definable subtypes of chronic obstructive pulmonary disease: a statement of the Fleischner Society. Radiology. 2015;277(1):192-205.

14. Smith BM, et al. Emphysema detected on computed tomography and risk of lung cancer: a systematic review and meta-analysis. Lung Cancer. 2012;77(1):58-63.

15. Xie $X$, et al. Morphological measurements in computed tomography correlate with airflow obstruction in chronic obstructive pulmonary disease: systematic review and meta-analysis. Eur Radiol. 2012;22(10):2085-93.

16. Kahnert K, et al. Relationship between clinical and radiological signs of bronchiectasis in COPD patients: results from COSYCONET. Respiratory Med. 2020;172:106117.

17. Karch A, et al. The German COPD cohort COSYCONET: aims, methods and descriptive analysis of the study population at baseline. Respir Med. 2016;114:27-37.
18. Jones P, Quirk F, Baveystock C. The St George's respiratory questionnaire. Respir Med. 1991;85:25-31.

19. Graham BL, et al. DLCO: adjust for lung volume, standardised reporting and interpretation. Eur Resp J. 2017;50(2):1701144.

20. Quanjer PH, et al. Multi-ethnic reference values for spirometry for the 3-95-yr age range: the global lung function 2012 equations. Eur Respiratory Soc. 2012;40:1324.

21. Stanojevic S, et al. Official ERS technical standards: Global Lung Function Initiative reference values for the carbon monoxide transfer factor for Caucasians. Eur Resp J. 2017;50(3):1700010.

22. Kellerer C, et al. Capnovolumetry in combination with clinical history for the diagnosis of asthma and COPD. NPJ Prim Care Resp Med. 2020;30(1):1-9.

23. Alfaro E, Gámez M, García N. Ensemble classification methods with applications in R. New Jersey: Wiley Online Library; 2019.

24. Alfaro E, Gámez M, Garcia N. adabag: an R package for classification with boosting and bagging. J Stat Softw. 2013;54(2):1-35.

25. Rätsch G, Onoda T, Müller K-R. Soft margins for AdaBoost. Mach Learn. 2001;42(3):287-320.

26. Kuhn M. Building predictive models in R using the caret package. J Stat Softw. 2008;28(5):1-26.

27. Hersh CP, et al. Non-emphysematous chronic obstructive pulmonary disease is associated with diabetes mellitus. BMC Pulm Med. 2014;14(1):164.

28. Wilson DO, et al. Association of radiographic emphysema and airflow obstruction with lung cancer. Am J Respir Crit Care Med. 2008;178(7):738-44.

\section{Publisher's Note}

Springer Nature remains neutral with regard to jurisdictional claims in published maps and institutional affiliations.
Ready to submit your research? Choose BMC and benefit from:

- fast, convenient online submission

- thorough peer review by experienced researchers in your field

- rapid publication on acceptance

- support for research data, including large and complex data types

- gold Open Access which fosters wider collaboration and increased citations

- maximum visibility for your research: over $100 \mathrm{M}$ website views per year

At $\mathrm{BMC}$, research is always in progress.

Learn more biomedcentral.com/submissions 